\title{
Gas-Cooled Thorium Reactor with Fuel Block of the Unified Design
}

\author{
Igor Shamanin, Sergey Bedenko, Yuriy Chertkov, and Ildar Gubaydulin
}

National Research Tomsk Polytechnic University, 30 Lenin Avenue, Tomsk 634050, Russia

Correspondence should be addressed to Sergey Bedenko; bedenko@tpu.ru

Received 5 June 2015; Revised 11 August 2015; Accepted 16 August 2015

Academic Editor: Amritendu Roy

Copyright ( 2015 Igor Shamanin et al. This is an open access article distributed under the Creative Commons Attribution License, which permits unrestricted use, distribution, and reproduction in any medium, provided the original work is properly cited.

\begin{abstract}
Scientific researches of new technological platform realization carried out in Russia are based on ideas of nuclear fuel breeding in closed fuel cycle and physical principles of fast neutron reactors. Innovative projects of low-power reactor systems correspond to the new technological platform. High-temperature gas-cooled thorium reactors with good transportability properties, small installation time, and operation without overloading for a long time are considered perspective. Such small modular reactor systems at good commercial, competitive level are capable of creating the basis of the regional power industry of the Russian Federation. The analysis of information about application of thorium as fuel in reactor systems and its perspective use is presented in the work. The results of the first stage of neutron-physical researches of a 3D model of the high-temperature gas-cooled thorium reactor based on the fuel block of the unified design are given. The calculation 3D model for the program code of MCU-5 series was developed. According to the comparison results of neutron-physical characteristics, several optimum reactor core compositions were chosen. The results of calculations of the reactivity margins, neutron flux distribution, and power density in the reactor core for the chosen core compositions are presented in the work.
\end{abstract}

\section{Introduction}

Nowadays in many countries nuclear power development programs are established. In the Russian Federation federal special-purpose program "New Generation Nuclear Power Technologies for 2010-2015 and Perspective to 2020" was adopted. The basis of this program is a new technological platform: closed nuclear fuel cycle (CNFC) and fast neutron reactors.

Researches aimed at developing nuclear technologies capable of including natural uranium and thorium into the nuclear fuel cycle correspond to the new technological platform. First of all these are the technologies of CNFC and fast neutron reactors (BN-600, BN-800, and BN-1200 reactor types) and innovative projects of perspective types of lowpower reactors and nuclear power reactors $(10-100 \mathrm{MWt})$.

In recent years, a great variety of research works in the field of low-power reactors has been presented. A small modular reactor on fast neutrons with sodium coolant created by Toshiba and ARC-100 construction promoted by Advanced
Reactor Concepts are considered to be the most developed projects.

In Russia, the demonstration block "BREST-300-OD," which will be put into operation at Siberian Chemical Combine by 2020, meets the well-developed concepts of lowpower fast reactors. It is expected to be a new link of the perspective model of CNFC in Russia [1].

Another development direction in a small modular niche is occupied by high-temperature and super high-temperature reactors. In this direction a number of designs and detailed schemes with temperatures from 900 to $1500 \mathrm{~K}$ were presented, high-temperature gas-cooled reactors being the most advanced [2-10].

It is obvious that low-power nuclear reactors are not an original idea. However, the projects on designing thorium fuel reactors on their basis are considered very perspective in the field of nuclear power industry. Besides, the market of low-power nuclear reactors with thorium fuel can be very advantageous in Russia. 
The purpose of the research is to develop the concept of a thorium low-power safe reactor based on the fuel block of the unified design.

It is supposed that during implementation of the concept all new available knowledge in the field of nuclear reactor industry and physics of neutrons will be applied. Development of the concept will allow starting a range of works which in their turn will result in the project of high-temperature gascooled thorium reactor (HTGTR) and further commercial realization.

It should be noted that in Russia nuclear technologies connected with commerciality of thorium low-power reactors are innovative, technically and economically are not investigated and are associated with significant financial investments.

\section{Analysis of Information Materials of the Use of Thorium as Nuclear Power Plants Fuel}

Due to calculation and experimental information (ENDSF (Evaluated Nuclear Structure Data File), EXFOR (Experimental Nuclear Reaction Data)) about neutron resonance absorption by thorium and uranium nuclei, one specific peculiarity can be reported. In the neutrons energy interval from 4 to $30 \mathrm{eV}$, when they interact with nuclei of U-238, two significantly powerful resonances with amplitudes of 11000 and 8000 barns (the cross section of absorption depends on the energy) can be observed. When neutrons interact with nuclei of Th-232 in the same energy interval, there is resonance of about 300 barns, but the amplitude is negligible in comparison with the resonances in the case with $\mathrm{U}$ 238. This peculiarity allowed explaining an important fact discovered in numerical experiments. When raw U-238 in oxide fuel composition $(\mathrm{U}, \mathrm{Pu}) \mathrm{O}_{2}$ was substituted by raw Th-232, optimum ratio of the water (moderator) volume to the fuel volume in a multiplying system from fuel pins with the diameter to $0.012 \mathrm{~m}$ increased significantly (5 times). Numerical experiments were performed at the Institute of Safety and Reactor Technologies Researches (ISR-2) of Forschungszentrum Juelich (Germany, 1998-1999). In the researches physics of WWER reactors operating in long and super long fuel-element lifetimes (up to 10 years) on thorium, highly enriched uranium and plutonium were studied [11].

In further numerical experiments [11-13] and experimental works (the works were carried out on the basis of research reactor IRT-T, unique id of the works: RFMEFI59114X0001) one peculiarity (anomaly) was determined by the dependence of resonance absorption on the ratio of the moderator volume to the fuel volume in multiplying thorium system $((\mathrm{m} \%$ $\left.\mathrm{U}, \mathrm{n} \% \mathrm{Th}) \mathrm{O}_{2},(\mathrm{~m} \% \mathrm{Pu}, \mathrm{n} \% \mathrm{Th}) \mathrm{O}_{2}\right)$, operating in thermal neutrons spectrum. The anomaly lies in the fact that at fixed value of the characteristic size of the nuclear fuel elements in a definite interval of changes of the moderator volume ratio to the fuel volume $\left(V_{\bmod } / V_{\text {fuel }}\right)$ there is sharp increase of the resonance neutrons absorption. In this interval the resonance absorption for uranium system exceeds absorption for plutonium by 3 and more times. Numerical experiments were carried out for multiplying systems with light-water and graphite moderators. Anomaly manifested in case of graphite at characteristic sizes of the fuel kernel (300-400) microns.

Much smaller resonance absorption in the case when raw nuclide is Th-232 provides two main advantages. The first one is that strong internal block-effect in distribution of epithermal neutrons flux distribution in the fuel kernel volume, which results in absorption of decelerating neutrons in relatively thin marginal layers, is much smaller in thorium systems. As a result integral fission quantity in nuclear fuel element increases at unchanged size. It provides great increase of fuel use efficiency, due to increase of lifetime as well. The second one is that in thorium system there is bigger amount of the moderator. It causes significant increase of thermal inertia with all ensuing advantages such as safety and reliability $[3,4,6,11-16]$.

The numerical researches and experiments, the results of which were presented above, were carried out for fuel compositions, plutonium, and highly enriched U-235 served as an igniter in start-up loading of the reactor core (RC). Thus physics of the fuel composition and reactors neither in thorium-uranium nor in uranium-plutonium NFC was studied. In case of ( $\mathrm{m} \% \mathrm{Pu}, \mathrm{n} \% \mathrm{Th}) \mathrm{O}_{2}$ composition it was thorium-plutonium NFC with the maximum possible burnup of fissionable nuclides of uranium, and plutonium formed and was burnable during nuclear fuel lifetime.

The results and the peculiarities of neutrons resonance absorption obtained in multiplying grids with thorium point out the necessity to change traditional design solutions for the grids, if the raw nuclide is Th-232.

The works performed within the framework of three state contracts (state contract number P777, 2010; agreement number 14.B37.21.0473, 2012; agreement number 14411.9990019.05110, 2014) allowed reviewing the decision for multiplying grids with thorium. Optimization of geometrical characteristics of fuel assemblies and nuclear fuel composition, particles size, and coatings of microcapsulated fuel (microfuel) dispersed into graphite of the fuel blocks of HTGTR operating with the maximum burn-up was made. The received scientific results and solutions made it possible to start complex neutron-physical calculations of HTGTR.

\section{Calculation Model of the High-Temperature Gas-Cooled Thorium Nuclear Power Reactor}

Design solutions accepted for HTGTR and neutron-physical characteristics necessary for comparison determined the reactor calculation model and the degree of $\mathrm{RC}$ elements specification.

Calculation model of HTGTR was created using MCU-5 program [17]. Geometrical module of MCU-5 allows simulating 3D systems with different complexity geometry using combinatorial approach based on description of complicated systems by combinations of elementary bodies and surfaces.

Detailed models of fuel pellets (FP) with heterogeneous fuel kernels, fuel assemblies, and RC of HTGTR were created. The temperature of all elements of the $\mathrm{RC}$ and core reflector was considered to be $1200 \mathrm{~K}$. Realized in MCU possibilities of 


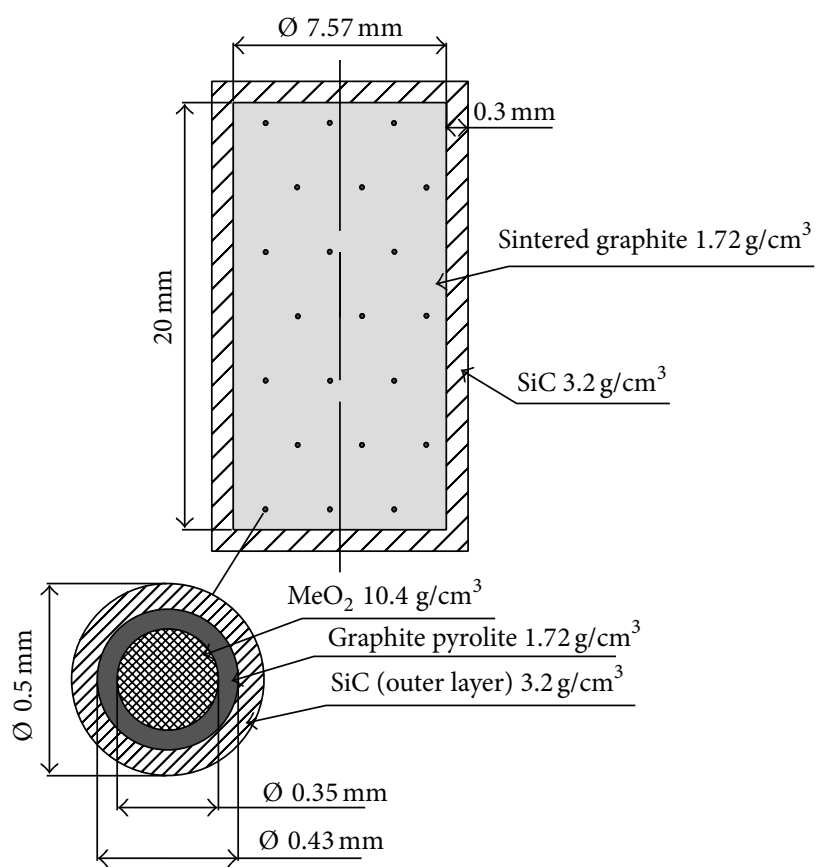

(a)

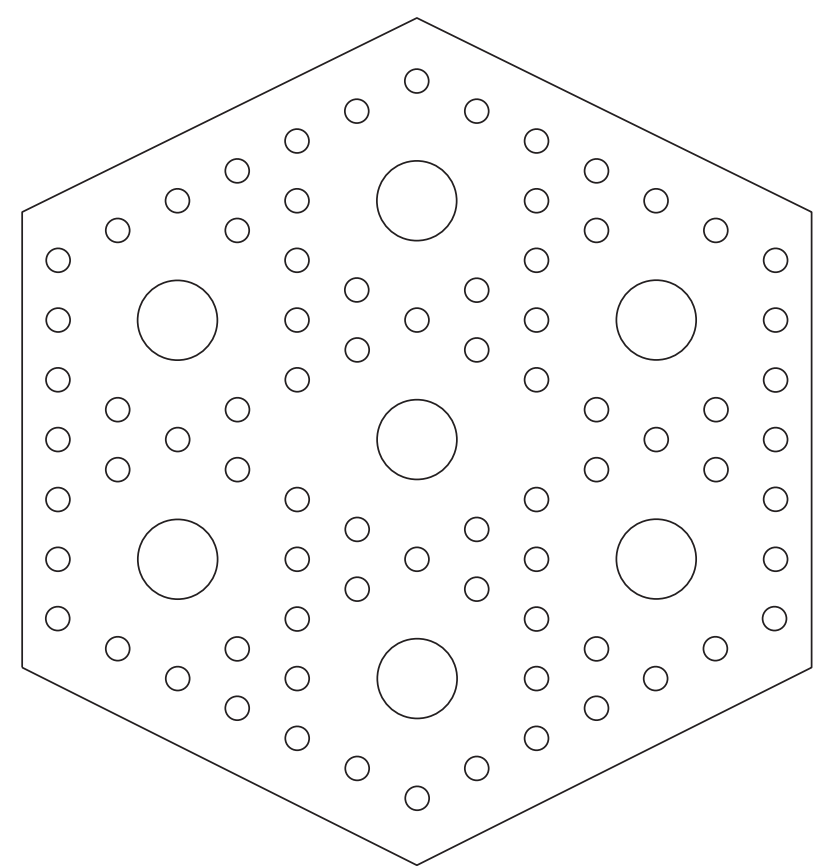

(b)

FIGURE 1: Calculation model of HTGTR fuel assembly: (a) 0500 and 1000 type fuel pellet of HTGTR; (b) HTGTR fuel assembly.

TABLE 1: Classification of FPs of HTGTR.

\begin{tabular}{lcccc}
\hline Fuel pellet type XXXX & Fuel volume $\left(\mathrm{MeO}_{2}\right)\left(\mathrm{m}^{3}\right)$ & Graphite volume $\left(\mathrm{m}^{3}\right)$ & SiC volume, $\left(\mathrm{m}^{3}\right)$ & Heavy metal mass $(\mathrm{kg})$ \\
\hline 0500 & $1.122 \cdot 10^{-8}$ & $8.770 \cdot 10^{-7}$ & $1.917 \cdot 10^{-7}$ & $0.103 \cdot 10^{-3}$ \\
1000 & $2.245 \cdot 10^{-8}$ & $8.539 \cdot 10^{-7}$ & $2.036 \cdot 10^{-7}$ & $0.206 \cdot 10^{-3}$ \\
2000 & $4.489 \cdot 10^{-8}$ & $1.949 \cdot 10^{-6}$ & $3.360 \cdot 10^{-7}$ & $0.411 \cdot 10^{-3}$ \\
\hline
\end{tabular}

describing complex geometrical objects, containing repeated elements with the help of grids, were used during the calculation model development.

Fuel pellet and fuel kernel: microfuel is a kernel from coated fissionable material dispersed into graphite matrix of cylindrical FPs, which are placed in the RC of HTGTR. Pu and Th-232 in equal proportions were used as fissionable material (heavy metal). Microfuel had bilayered coatings: layer 1: pyrolytic carbon (PyC) with the density of $1720 \mathrm{~kg} / \mathrm{m}^{3}$; layer 2: silicon carbide ( $\mathrm{SiC}$ ) with the density of $3210 \mathrm{~kg} / \mathrm{m}^{3}$. Configurations of the fuel kernel, coatings, and fuel pellets (see Figure 1) were chosen on the basis of scientific results obtained in [3, 4, 6, 11-16].

Preliminary estimates of energy production efficiency showed that bilayered coating decreases the fuel cost component considerably. Safety barrier is created by $\mathrm{SiC}$ coating on the fuel pellet surface.

Taking into account that coatings of microfuel are made from materials, in which cross sections do not have significant resonance peaks, the method of partial homogenization was used. In this approximation the fuel kernel coatings homogenize with the graphite matrix filling new volume with new concentrations. Acceptability of homogenization was testified in the works of the authors in $[5,7,17-$ 21]. Homogenization of the fuel kernel is an impermissible approximation and it immediately causes noticeable deviation of $k_{\text {eff }}$ (effective multiplication coefficient) (to 7-8\%) from more precise simulation $[5,7,20,21]$ and experimental results $[5,19]$.

Three types of FPs of HTGTR were considered in the calculations. FPs of 0500 and 1000 type have the following constructional characteristics (see Figure 1(a)): diameter of $8.17 \cdot 10^{-3} \mathrm{~m}$ (with SiC surface layer); height of $2.06 \cdot 10^{-2} \mathrm{~m}$ (with $\mathrm{SiC}$ surface layer); volume of $1.08 \cdot 10^{-6} \mathrm{~m}^{3}$.

FPs of 2000 type have the following constructional characteristics: diameter of $1.2 \cdot 10^{-2} \mathrm{~m}$ (with $\mathrm{SiC}$ surface layer); height of $2.06 \cdot 10^{-2} \mathrm{~m}$ (with $\mathrm{SiC}$ surface layer); volume of $2.33 \cdot 10^{-6} \mathrm{~m}^{3}$. Close classification of the considered FPs is presented in Table 1.

Depending on the heavy metal composition in the FPs, the fuel assemblies of XXXXB and XXXXZ type were considered (see Figure 1(b)).

The fuel assembly of XXXXB type (of the first type) is $\mathrm{Pu}$, $10 \%$; Th-232, 90\%. The fuel assembly of XXXXZ type (of the second type) is $\mathrm{Pu}, 50 \%$; Th-232, 50\%. The modifications of isotope composition of $\mathrm{Pu}$ in FPs are given in Table 2. 
TABLE 2: Isotope composition of Pu in FPs of HTGTR.

\begin{tabular}{|c|c|c|c|c|c|}
\hline $\begin{array}{l}\text { Pu isotope composition } \\
\text { modification }\end{array}$ & $\mathrm{Pu}-238(\%)$ & $\mathrm{Pu}-239$ (\%) & $\mathrm{Pu}-240(\%)$ & $\mathrm{Pu}-241(\%)$ & $\mathrm{Pu}-242(\%)$ \\
\hline 1 & 1.8 & 59 & 23 & 12.2 & 4 \\
\hline 2 & 0 & 94 & 5 & 1 & 0 \\
\hline
\end{tabular}

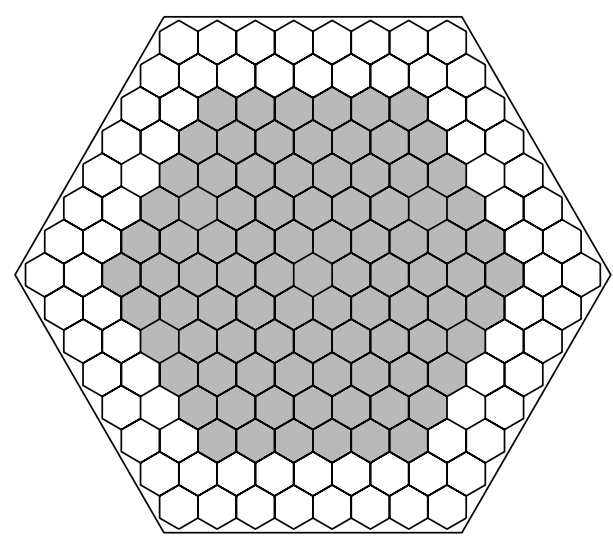

(a)

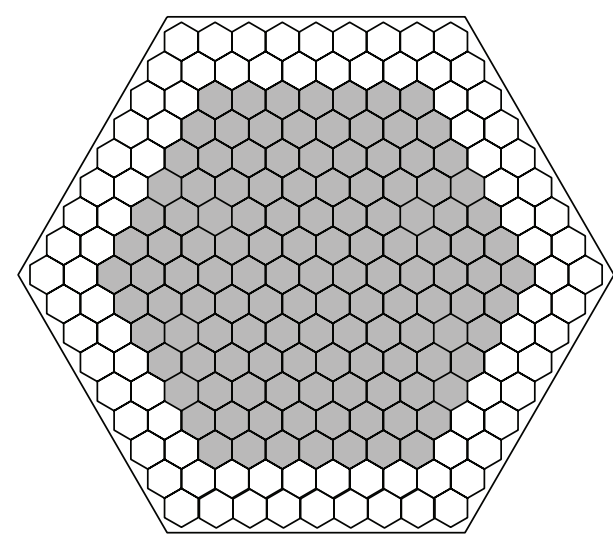

(b)

FIGURE 2: Calculation model of HTGTR reactor core: (a) small reactor core with the reflector; (b) large reactor core with the reflector.

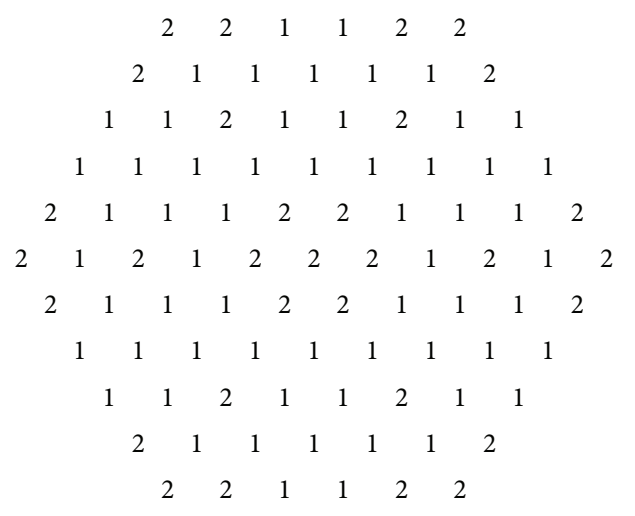

(a)

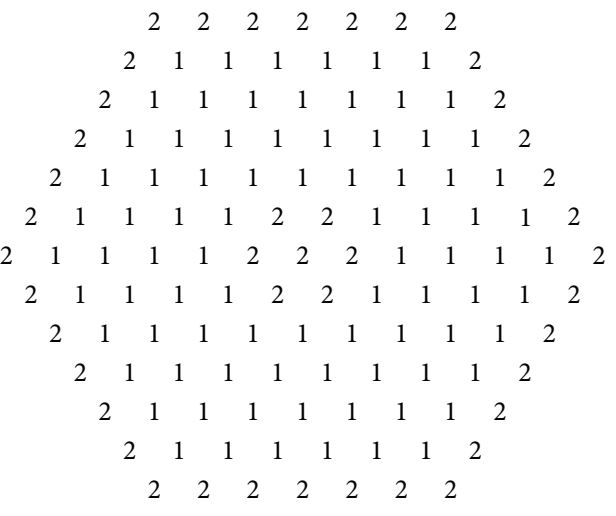

(b)

Figure 3: Arrangement schemes of fuel assemblies of the first and second type for small and large reactor cores: (a) small reactor core; (b) large reactor core.

Example. Fuel assembly 0500Z2 is a fuel assembly of the second type which consists of FPs of 0500 type (see Table 1 ) and has the second isotope composition of $\mathrm{Pu}$ (see Table 2). FP: fuel volume $\left(\mathrm{MeO}_{2}\right)$ is equal to $1.122 \cdot 10^{-8} \mathrm{~m}^{3}$; graphite volume is $8.770 \cdot 10^{-7} \mathrm{~m}^{3}$; $\mathrm{SiC}$ volume equals $1.917 \cdot 10^{-7} \mathrm{~m}^{3}$; heavy metal mass is $0.103 \cdot 10^{-3} \mathrm{~kg}$. Percentage of heavy metal, $\%$, is $\mathrm{Pu}, 50$; Th-232, 50. Isotope composition of $\mathrm{Pu}(\%)$ is 238 , $0 ; 239,94 ; 240,5 ; 241,1 ; 242,0$.

Fuel Assembly (Fuel Block of the Unified Construction). It is a hexagon graphite fuel block with the width across the flats of $0.2 \mathrm{~m}$ and height of $0.8 \mathrm{~m} ; 78$ holes with the diameter of 8.2 $\cdot 10^{-3} \mathrm{~m}$ for FPs of 0500 and/or 1000 type; 7 holes with the diameter of $2.4 \cdot 10^{-2} \mathrm{~m}$ for passing gaseous coolant (helium) (see Figure 1(b)).
Reactor Core. Cores are loaded with specified fuel assemblies by two methods (see Figures 2 and 3):

(1) Small reactor core consists of fuel assemblies with fuel-containing parts, each about $2.4 \mathrm{~m}$ long, surrounded by two graphite blocks without holes; at the top and the bottom the reactor core is covered by $0.3 \mathrm{~m}$ thick graphite.

(2) Large reactor core consists of fuel assemblies with fuel-containing parts, each about 2.0 or $2.4 \mathrm{~m}$ long, surrounded by two rows of graphite blocks without holes; at the top and at the bottom the reactor core is surrounded by $0.3 \mathrm{~m}$ thick graphite. 
TABLE 3: Calculation results of effective multiplication coefficient $k_{\text {eff }}$ and reactivity margin of the calculated type of HTGTR.

\begin{tabular}{|c|c|c|c|c|c|c|}
\hline Number & Reactor core height (m) & Reactor core type & Fuel pellets type & Isotope composition of $\mathrm{Pu}$ & $k_{\mathrm{eff}}$ & $\Delta k / k(\%)$ \\
\hline 1 & 2 & Large & 1000 & 1 & 1.0591 & 5.57 \\
\hline 2 & 2 & Large & 1000 & 2 & 1.2445 & 19.65 \\
\hline 3 & 2 & Large & 500 & 1 & 0.9611 & -4.06 \\
\hline 4 & 2 & Large & 500 & 2 & 1.1243 & 11.06 \\
\hline 5 & 2.4 & Small & 1000 & 1 & 1.0611 & 5.75 \\
\hline 6 & 2.4 & Small & 1000 & 2 & 1.2471 & 19.81 \\
\hline 7 & 2.4 & Small & 500 & 1 & 0.9785 & -2.20 \\
\hline 8 & 2.4 & Small & 500 & 2 & 1.1475 & 12.85 \\
\hline 9 & 2.4 & Large & 1000 & 2 & 1.2665 & 21.49 \\
\hline 10 & 2.4 & Large & 500 & 2 & 1.1612 & 13.76 \\
\hline 11 & 2.4 & Large & 1000 & 1 & 1.0801 & 7.80 \\
\hline 12 & 2.4 & Large & 500 & 1 & 0.9921 & -1.52 \\
\hline
\end{tabular}

\section{Neutron-Physical Calculations of High- Temperature Gas-Cooled Thorium Reactor}

Neutron-physical calculations were carried out using the calculation code of MCU created in Russian National Research Center "Kurchatov Institute." MCU program is intended for precision simulation of the processes of neutrons and photons transfer by analogue and nonanalogue methods of Monte-Carlo on the basis of evaluated nuclear data in any reactor types, considering changes of reactor material isotope composition during the lifetime [17].

BURNUP module was used for calculating the reactor lifetime. The module is a part of the program MCU5 and is intended for calculating composition changes of the reactor fissile isotopes and absorbing materials during its lifetime. The module provides possibility of calculation prediction of reactor materials nuclide composition and multiplying properties of the reactor depending on the fuel burn-up during its operation. Calculation of isotope composition changes during the reactor lifetime was performed at the given dependence of medium power in the system on time.

Constant MCU5 package is based on nuclear data bank MCUDB50. Library ACE/MCU being a part of MCUDB50 is point-to-point presented neutron-physical constants received from the files of evaluated nuclear data (ENDF/B-VII.0, JEFF-3.1.1, and JENDL-4.0) using the international program NJOY-99. Library BNAB/MCU (a part of MCUDB50) is an extended and modified version of 26-group constants system BNAB-78. Other sources included into nuclear data bank MCUDB50 are Russian (Russian National Library of Evaluated Neutron Data, Resonant Parameters Library, Moderators Generalized Phonon Spectrum Library, Library of Evaluated Neutron Data, etc.) and available international libraries (ENDSF, EXFOR).

Thus nuclear data bank MCUDB50 comprises constants obtained in group and point-to-point approximation, which allowed using in calculations statistic algorisms based on Monte-Carlo method and multigroup approximations using subgroup parameters in calculations of group coefficients in decomposed Boltzmann equation.
The schemes of fuel assemblies arrangement (of the first and second types) for small and large reactor cores of HTGTR are presented in Figure 3.

Results of $k_{\text {eff }}$ calculation using MCU-5 for all considered calculation variants of HTGTR are presented in Table 3.

Calculation variants with the first isotope composition of $\mathrm{Pu}$ have the least values of $k_{\text {eff }}$ from all variants; thus they were excluded from any further consideration.

Calculation variants 2, 6, and 9 of the high-temperature gas-cooled thorium reactor with fuel assemblies of 1000B2 and 1000Z2 type (see Table 3) are the reactor with small and large cores with fuel assemblies of the first and second types, fuel pellets of 1000 type having the second isotope composition of $\mathrm{Pu}$ (see Tables 1 and 2).

The distributions of neutron fluxes and power densities were obtained for the selected variants; irregularity coefficient estimation was carried out.

Irregularity coefficient of neutron flux distribution is 1.5 for large reactor cores (calculation variants 2 and 9) and $\sim 1.4$ for small reactor core (calculation variant 6). Axial irregularity coefficient of power density for the reactor core with the height of $2 \mathrm{~m}$ (calculation variant 2 ) is $\sim 1.25$, and for the reactor core with the height of $2.4 \mathrm{~m}$ (calculation variants 6 and 9) it is $\sim 1.31$. Radial distribution irregularity coefficient does not exceed the value of $\sim 3$.

For calculation modification 9, that is, for HTGTR large reactor core with fuel assemblies of 1000B2 and 1000Z2 type, the reactivity margin is $21.49 \%$. When the reactor operates on the power of $60 \mathrm{MWt}$, the steady-state poisoning effect is $\sim 3.5 \%$ and the reactivity loss rate is $\sim 0.123 \%$ per day. It allows evaluating the reactor lifetime which is not more than 150 days.

In case of the small reactor core (calculation variant 2 ) the reactivity margin has a slightly smaller value $(\sim 20 \%)$, but due to significantly smaller sizes the reactivity loss rate is higher compared to the large reactor core; consequently, the reactor lifetime does not exceed 110 days.

The calculations showed that the modification, when the reactor core consists of fuel assemblies of the same type (e.g., 1000Z1 and 1000Z2 type) containing equal amount of Th-232 and $\mathrm{Pu}$, is considered more perspective. Initial 


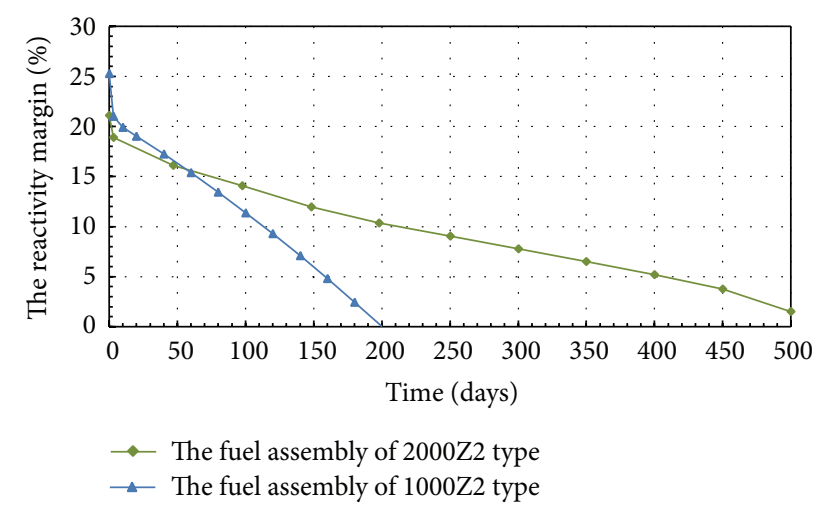

FIGURE 4: Change of the reactivity margin of HTGTR with fuel assemblies of $1000 \mathrm{Z} 2$ and $2000 \mathrm{Z} 2$ type.

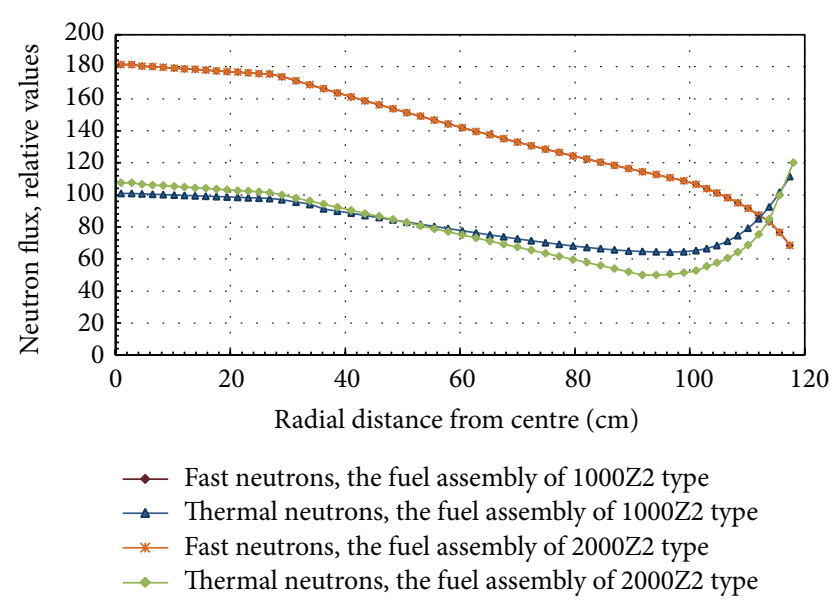

FIgURE 5: Radial flux density distribution of fast and thermal neutrons in the large core.

reactivity margin of HTGTR with fuel assemblies of $1000 \mathrm{Z} 2$ type increases up to $25.3 \%$, and the reactor lifetime continues up to 210 days (see Figure 4).

Such reactor core loading allowed reducing power and neutrons flux distribution tilts in comparison with earlier calculation variants (see Figures 5 and 6). Approximately $1.7 \%$ of Th-232 burns up in the reactor with fuel assembly of $1000 \mathrm{Z} 2$ type; accumulation of $\mathrm{U}-233$ is $\sim 10 \%$ of this amount (considering the burn-up of the latter).

One more way of creating HTGTR was an attempt to increase fuel loading. Fuel of 2000 type was used (see Table 4).

Such fuel is used for arranging the biggest RC (with 381 fuel assemblies of 2000Z2 type) with the height of $2.4 \mathrm{~m}$. The mass of plutonium in such RC increases by $\sim 4$ times, the initial reactivity margin of HTGTR being $21.1 \%$, while the rate of reactivity loss decreases to $0.035 \%$ per day and lifetime increases to 510 effective days (see Figure 4).

Figures 5 and 6 show the distribution of power density and neutron flux in large reactor core with fuel assemblies of $2000 \mathrm{Z} 2$ type radially.

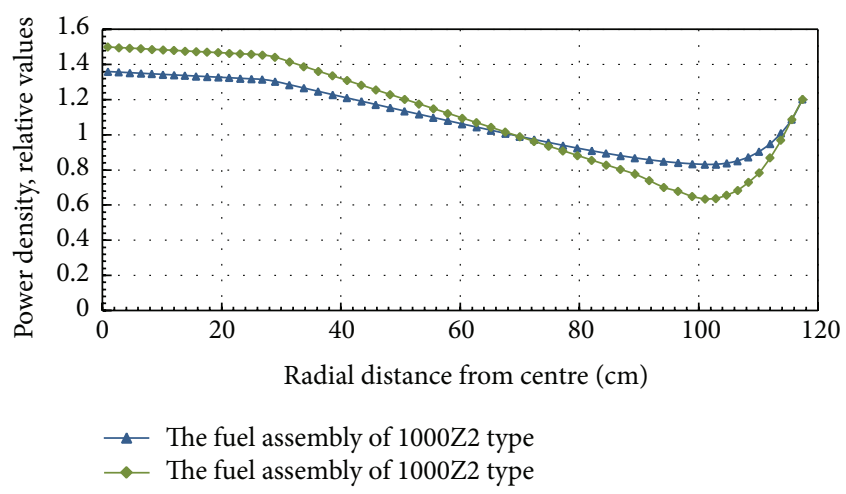

FIGURE 6: Radial power density distribution in the large core.

TABLE 4: Basic design parameters of the reactor.

\begin{tabular}{|c|c|}
\hline Performance & Value \\
\hline Full thermal reactor power (MWt) & 60 \\
\hline \multicolumn{2}{|l|}{ Neutrons flux density: } \\
\hline Thermal $\left(\mathrm{n} / \mathrm{cm}^{2} / \mathrm{s}\right)$ & $2.8 \cdot 10^{-13}$ \\
\hline Fast $\left(\mathrm{n} / \mathrm{cm}^{2} / \mathrm{s}\right)$ & $4.8 \cdot 10^{-13}$ \\
\hline \multicolumn{2}{|l|}{ Reactor geometrical parameters: } \\
\hline $\begin{array}{l}\text { Equivalent diameter of reactor core with reflector } \\
(\mathrm{m})\end{array}$ & 3.1 \\
\hline Height of reactor core with reflector (m) & 3.0 \\
\hline \multicolumn{2}{|l|}{ Reactor core geometrical parameters: } \\
\hline Reactor core equivalent diameter (m) & 2.4 \\
\hline Reactor core height (m) & 2.4 \\
\hline Fuel assembly type (fuel block of the unified design) & $2000 \mathrm{Z} 2$ \\
\hline Number of fuel assemblies in the reactor core (pcs) & 381 \\
\hline \multicolumn{2}{|l|}{ Fuel assembly geometrical parameters: } \\
\hline Width across the flats (m) & 0.2 \\
\hline Height (m) & 0.8 \\
\hline Fuel pellets number (pcs) & 3120 \\
\hline Heavy metal mass (kg) & 1.282 \\
\hline \multicolumn{2}{|l|}{ Fuel pellet geometrical parameters: } \\
\hline Height $(\mathrm{m})$ & 0.020 \\
\hline Diameter $(\mathrm{m})$ & 0.012 \\
\hline Heavy metal mass $(\mathrm{kg})$ & $0.411 \cdot 10^{-3}$ \\
\hline \multicolumn{2}{|l|}{ Full starting core fuelling: } \\
\hline Mass of Th (kg) & 244 \\
\hline Mass of $\mathrm{Pu}(\mathrm{kg})$ & 244 \\
\hline Maximum reactivity margin $(\Delta k / k), \%$ & 21.3 \\
\hline Full-power reactor operation time (days) & 510 \\
\hline
\end{tabular}

\section{Conclusion}

Several optimum arrangements of the reactor cores (calculation variants 2, 6, and 9) intended for low-power industry and creation of mobile nuclear power installations with the capacity to $60 \mathrm{MWt}$ were chosen from tens of HTGTR calculation variants. 
Calculation variants 2, 6, and 9 of HTGTR with the fuel assemblies of 1000B2 and 1000Z2 type (see Table 3) are a reactor with small and large reactor cores with fuel assemblies of the first and second type and fuel pellets of 1000 type, which have the second isotopic composition of Pu (see Table 2).

The main disadvantage of these modifications of HTGTR is that $\mathrm{Pu}-239$ does not manage to burn up and U-233 to accumulate to appreciable amount during short reactor lifetime (150 days for the large reactor core and 110 days for small reactor cores).

The calculations showed that the most perspective solution is when the reactor core consists of fuel assemblies of the same type and contains equal amount of Th-232 and Pu. In this case initial reactivity margin for the large reactor core loaded with fuel assemblies of 1000Z2 type increases up to $25.3 \%$ and the lifetime increases to 210 effective full power days (see Figure 4). During 210 days, 1.7\% of Th-232 burns up in the reactor with fuel assembly of $1000 \mathrm{Z} 2$ type; accumulation of U-233 is $\sim 10 \%$ of this amount (considering the burn-up of the latter). At such loading the distributions of flux density and power density become uniform over the whole reactor (see Figures 5 and 6).

One more way of creating HTGTR was an attempt to increase fuel loading. Fuel of 2000 type was used. Such fuel is used for arranging the biggest reactor core (with 381 fuel assemblies) with the height of $2.4 \mathrm{~m}$. The mass of plutonium in such reactor core increases by $\sim 4$ times, the initial reactivity margin of HTGTR being $21.1 \%$, while the rate of reactivity loss decreases to $0.035 \%$ per day and lifetime increases to 510 effective days (see Figure 4).

It should be noted that only the first stage of neutronphysical calculations researching the possibility of creating thorium reactor has been performed. In the calculation model of the reactor the control rods were not considered.

\section{Conflict of Interests}

The authors declare that there is no conflict of interests regarding the publication of this paper.

\section{Acknowledgments}

The research was performed at the support of Board for Grants of the President of the Russian Federation, competition for young scientists and postgraduate students for getting the presidential scholarship, Grant no. SP-295.2015.2. Experimental researches for verification of calculations were carried out at IRT-T at the financial support of the Ministry of Education and Science of the Russian Federation (works unique identifier RFMEFI59114X0001), Agreement no. 14.591.21.0001 of 15.08.2014.

\section{References}

[1] E. F. Seleznev, Fast Breeder Reactor Kinetics, Nauka, Moscow, Russia, 2013, (Russian).

[2] A. I. Kiryushin, N. N. Ponomarev-Stepnoi, and E. S. Glushkov, "Project of the GM-MHR high-temperature helium reactor with gas turbine," Nuclear Engineering and Design, vol. 173, pp. 119-129, 1997.

[3] H. Nabielek, W. Kühnlein, W. Schenk, W. Heit, A. Christ, and H. Ragoss, "Development of advanced HTR fuel elements," Nuclear Engineering and Design, vol. 121, no. 2, pp. 199-210, 1990.

[4] N. N. Ponomarev-Stepnoi, N. G. Abrosimov, A. V. Vasyaev et al., "Similarity of high-temperature gas-cooled reactor technologies and designs in Russia and USA," Atomic Energy, vol. 108, no. 2, pp. 89-96, 2010 (Russian).

[5] N. G. Kodochigov, J. P. Suharev, E. V. Marova et al., "Analytical and experimental study of the GT-MGR core neutronic characteristics," Atomic Energy, vol. 102, no. 1, pp. 63-68, 2007 (Russian).

[6] M. Fu, T. Liang, Y. Tang, X. U. Zhichang, and C. Tang, "Preparation of $\mathrm{UO}_{2}$ kernel for HTR-10 fuel element," Journal of Nuclear Science and Technology, vol. 41, no. 9, pp. 943-948, 2004.

[7] V. Şeker and Ü. Çolak, "HTR-10 full core first criticality analysis with MCNP," Nuclear Engineering and Design, vol. 222, no. 2-3, pp. 263-270, 2003.

[8] M. Miletić, R. Fukač, I. Pioro, and A. Dragunov, “Development of gas cooled reactors and experimental setup of high temperature helium loop for in-pile operation," Nuclear Engineering and Design, vol. 276, pp. 87-97, 2014.

[9] R. Stainsby, K. Peers, C. Mitchell, C. Poette, K. Mikityuk, and J. Somers, "Gas cooled fast reactor research in Europe," Nuclear Engineering and Design, vol. 241, no. 9, pp. 3481-3489, 2011.

[10] W. J. Lee, Y. W. Kim, and J. Chang, "Perspectives of nuclear heat and hydrogen," Nuclear Engineering and Technology, vol. 41, no. 4, pp. 412-426, 2009.

[11] I. V. Shamanin, A. A. Ukhov, H. J. Rutten, K. A. Haas, and W. Sherer, The Use of $\left(\right.$ Th, U,Pu) $\mathrm{O}_{2}$ Fuel in a Water Water Energy Reactor: Physics and Fuel Cycle Simulation by Means of the V.S.O.P. (97) Computer Code, Forschungszentrum Juelich $\mathrm{GmbH}$, Institute for Safety Research and Reactor Technology, Jülich, Germany, 1999.

[12] I. Shamanin, S. Bedenko, and I. Gubaydulin, "Advantages of thorium nuclear fuel for thermal-neutron reactor," Advanced Materials Research, vol. 1084, pp. 275-279, 2015.

[13] A. Talamo and W. Gudowski, "Performance of the gas turbinemodular helium reactor fuelled with different types of fertile TRISO particles," Annals of Nuclear Energy, vol. 32, no. 16, pp. 1719-1749, 2005

[14] H.-J. Allelein, M. J. Kania, H. Nabielek, and K. Verfondern, "Thorium fuel performance assessment in HTRs," Nuclear Engineering and Design, vol. 271, pp. 166-170, 2014.

[15] C. Tang, B. Liu, Z. Li, Y. Quan, H. Zhao, and Y. Shao, "SiC performance of coated fuel particles under high-temperature atmosphere of air," Nuclear Engineering and Design, vol. 271, pp. 64-67, 2014.

[16] M. J. Kania, H. Nabielek, K. Verfondern, and H.-J. Allelein, "Testing of HTR $\mathrm{UO}_{2}$ TRISO fuels in AVR and in material test reactors," Journal of Nuclear Materials, vol. 441, no. 1-3, pp. 545562, 2013.

[17] D. S. Oleynik, D. A. Shkarovskiy, E. A. Gomin et al., "The status of MCU-5," Physics of Atomic Nuclei, vol. 75, no. 14, pp. 16341646, 2012.

[18] R. Kelly and D. Ilas, "Verification of a depletion method in SCALE for the Advanced High-Temperature Reactor," Nuclear Technology, vol. 183, no. 3, pp. 391-397, 2013.

[19] V. P. Garin, A. E. Glushkov, E. S. Glushkov et al., "Evaluated benchmark experiments in critical assemblies modeling HTGR 
peculiarities at the Astra facility," Questions of Atomic Science and Technics. Physics of Nuclear Reactors, vol. 2, pp. 75-96, 2009 (Russian).

[20] B. K. Chukbar, "Verification of statistical method CORN for modeling of microfuel in case of high grain concentration," Questions of Atomic Science and Technics. Physics of Nuclear Reactors, no. 1-2, pp. 15-21, 2014 (Russian).

[21] R. Plukiene and D. Ridikas, "Modelling of HTRs with Monte Carlo: from a homogeneous to an exact heterogeneous core with microparticles," Annals of Nuclear Energy, vol. 30, no. 15, pp. 1573-1585, 2003. 

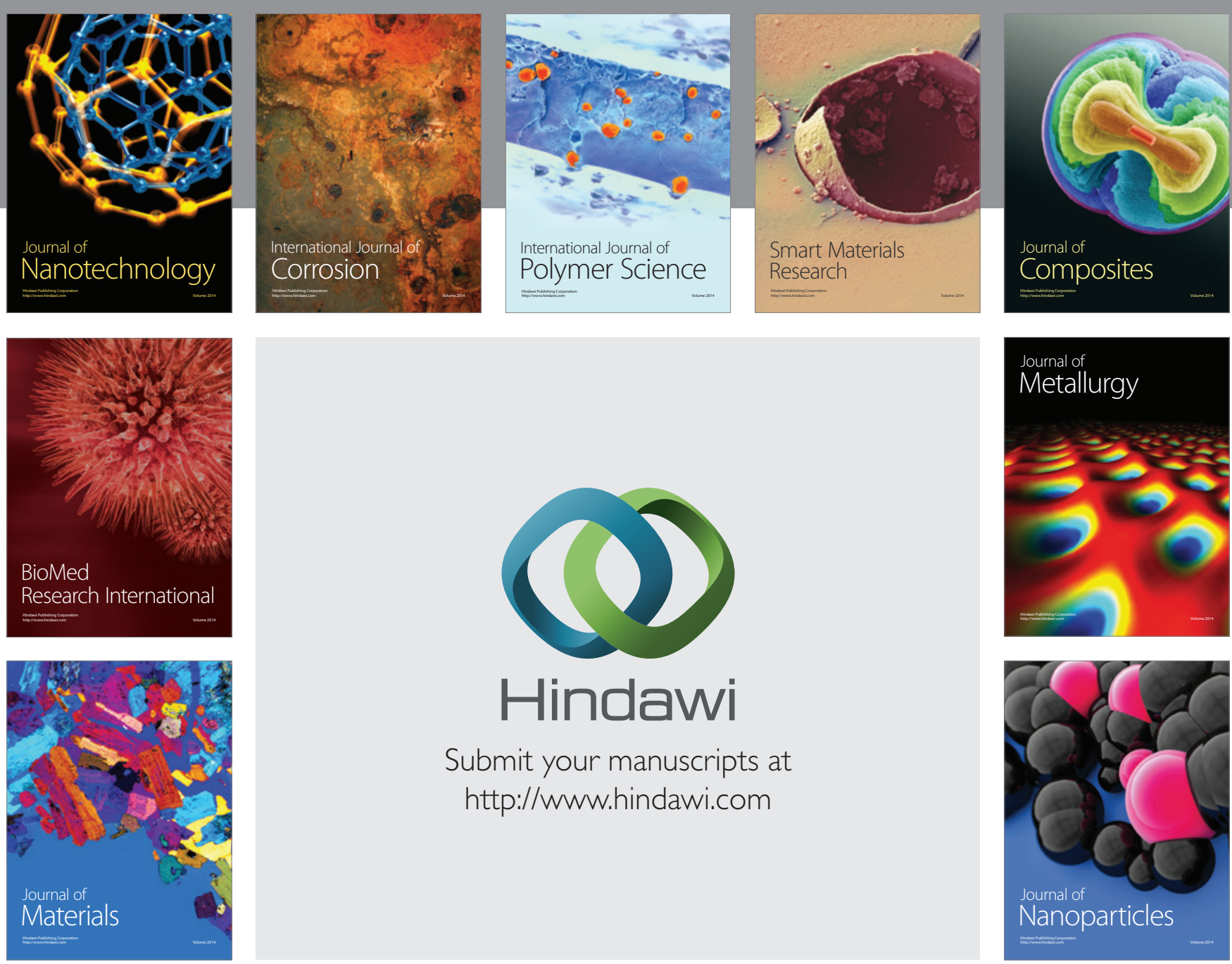

Submit your manuscripts at http://www.hindawi.com
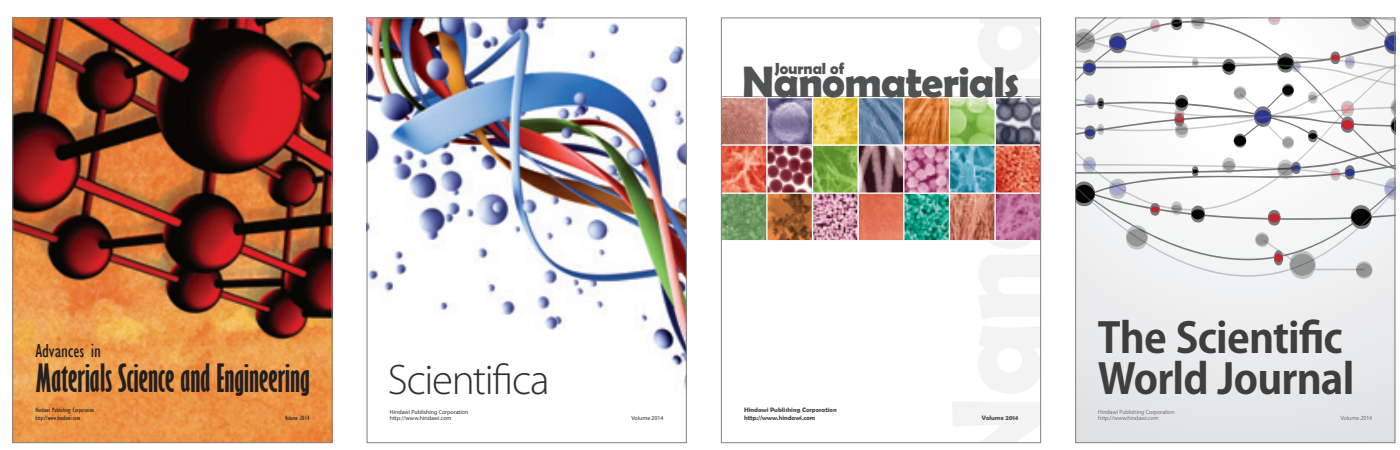

\section{The Scientific World Journal}
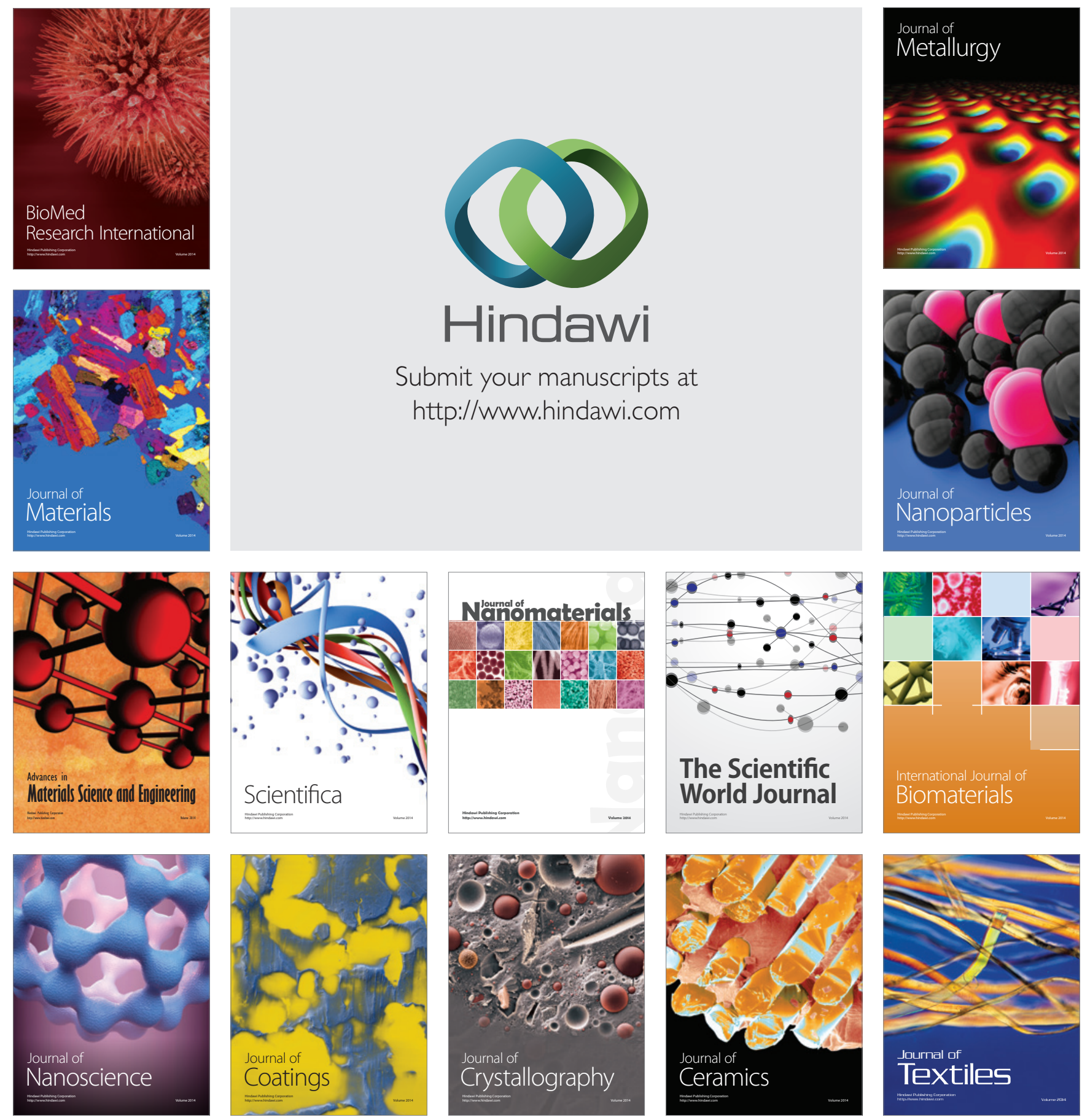